\title{
104. ANOTACIONES SOBRE LA VEGETACIÓN NITRÓFILA DEL ARCHIPIÉLAGO DE COLUMBRETES (CASTELLÓN)
}

\author{
Ana JUAN y Manuel B. CRESPO
}

Notes on the nitrophilous plant communities of the Columbretes Archipelago (Castellon, eastern Spain)

Palabras clave. Vegetación nitrófila, nomenclatura, Iberolevantina, España.

Key words. Nitrophilous plant communities, nomenclature, Iberolevantine, Spain.

El Parque Natural de las islas Columbretes se encuentra situado a unos $56 \mathrm{~km}$ al este de la costa de Castellón (España) y está formado por cuatro grupos de islotes. De todos ellos, sólo las islas de mayor tamaño -conocidas como isla Grossa, Ferrera, Foradada y Mancolibrepresentan una densa cubierta vegetal.

El origen de este archipiélago es volcánico y data de hace aproximadamente un millón de años (Aparicio et al., 1991). Sus características climáticas -bioclima termomediterráneo semiárido (cf. Rivas-Martínez \& Loidi, 1999a)y edáficas -suelos volcánicos, nitrificados por guano de aves marítimas y salinizados por el hálito marino-, hacen que su flora y su vegetación estén sometidas a un largo período de estrés, encontrándose el óptimo fenológico de la mayoría de los táxones y asociaciones durante el invierno (diciembre-marzo). Biogeográficamente, las Columbretes pertenecen al subsector Valenciano-Castellonense, sector Valenciano-Tarraconense, subprovincia Catalano-Valenciano -Provenzal, provincia Mediterráneo -Iberolevantina (RivasMartínez \& Loidi, 1999b).

Las peculiares características ecológicas del archipiélago, unidas al alto grado de antropización que algunas islas han sufrido durante los últimos cien años (e.g., isla Grossa), han provocado que la mayoría de las comunidades vegetales actuales sean nitrófilas y que en las proximidades de las construcciones humanas en la isla Grossa (e.g., las Casernas y el Faro) lleguen a dominar las formaciones hipernitrófilas. No obstante, la presencia de numerosas colonias de aves marinas contribuye a aumentar los niveles de nitrificación en el suelo, de modo que la existencia de algunas asociaciones en el archipiélago va ligada a la actividad de dichas aves.

En este trabajo fitosociológico, se revisan nomenclaturalmente algunas de las asociaciones de matiz nitrófilo descritas del archipiélago de las Columbretes y se describe una nueva subasociación endémica de éste. Los nombres de los táxones citados en el texto y tablas se ajustan, cuando no se indica explícitamente, a los que aparecen en Mateo \& Crespo (2001).

\section{Lavatero davaei-Suaedetum verae $\mathrm{O}$. Bolòs,}

Folch \& Vigo in O. Bolòs 1989

[Folia Bot. Miscel. 6: 121. Holotypus: Tabla 2, invent. 9]

(Suaedetum verae sensu Carretero \& Boira 1987, non Br.-Bl. ex O. Bolòs \& Molinier 1958; Lavatero davaei-Suaedetum verae $\mathrm{O}$. Bolòs, Folch \& Vigo ex Rivas-Martínez et al. 2001, nom. superfl.). (Tabla 1)

Matorral dominado por Suaeda vera Forssk., el cual se acompaña de elementos nitrófilos de óptimo en otras clases fitosociológicas (e.g., Stellarietea mediae Tüxen, Lohmeyer \& Preissing ex von Rochow 
Tabla 1

Lavatero davaei-Suadetum verae O. Bolòs, Folch \& Vigo in O. Bolòs 1989

(Carthamo-Salsolion oppositifoliae, Salsolo-Peganetalia, Pegano-Salsoletea)

\begin{tabular}{lccccccc}
\hline Inventario $\mathrm{n}^{\mathbf{0}}$ & 1 & 2 & 3 & 4 & 5 & 6 & 7 \\
Orientación e inclinación $\left({ }^{\circ}\right)$ & $20 \mathrm{SE}$ & $50 \mathrm{E}$ & $10 \mathrm{E}$ & $20 \mathrm{~S}$ & $30 \mathrm{~W}$ & $20 \mathrm{~W}$ & $3 \mathrm{NE}$ \\
Altitud $(\mathrm{m})$ & 36 & 22 & 24 & 24 & 34 & 22 & - \\
Cobertura $(\%)$ & 80 & 90 & 100 & 60 & 75 & 60 & 100 \\
Área $\left(\mathrm{m}^{2}\right)$ & 100 & 50 & 50 & 100 & 80 & 200 & - \\
Fecha (mes/año) & $4 / 94$ & $3 / 95$ & $3 / 95$ & $3 / 95$ & $3 / 95$ & $3 / 95$ & -
\end{tabular}

Caract. asociación y alianza

Suaeda vera

Lavatera davaei

4

2

5

4

$\begin{array}{llll}4 & 5 & 5 & 5.3 \\ + & & -5 & -1.1\end{array}$

Caract. de unidades superiores

Lobularia columbretensis

Sonchus dianae

Medicago citrina

\section{Compañeras}

Mesembryanthemum nodiflorum

Erodium chium

Asparagus horridus

Lavatera arborea

Chenopodium murale

Euphorbia terracina

Silene sclerocarpa

Sonchus asper

$\begin{array}{ll}1 & 1 \\ - & - \\ - & -\end{array}$

2

$\begin{array}{lllllll}- & 1 & - & + & + & + & - \\ - & - & + & 2 & - & - & + \\ + & + & - & 1 & - & - & - \\ - & - & - & - & 2 & 1 & - \\ - & - & - & + & - & + & - \\ - & - & + & - & - & - & + \\ - & - & - & - & + & + & - \\ - & - & + & - & - & - & +\end{array}$

Compañeras presentes en un solo inventario: En inv. 1: Brachypodium distachyon 1. En inv. 3: Lotus edulis + ; Medicago littoralis + . En inv. 4: Bromus hordeaceus 1. En inv. 5: Patellifolia patellaris 1. En inv. 7: Hymenolobus procumbens + ; Plantago coronopus + ; Silene sp. + ; Triplachne nitens + .

Localidades: 1.- Próximo a las Casernas, Grossa. 2.- Cercano al Cabo del Tabaco, Grossa. 3.- Cercano al Cementerio, Grossa. 4.- Cerca del Cementerio, en la ladera orientada hacia la Foradada, Grossa. 5.- y 6.Ferrera. 7.- Illa Grossa, prop del cementeri, sòl arenós (Holótipo: BoLòs, 1989).

1951, Artemisietea vulgaris Tüxen, Lohmeyer \& Preissing ex von Rochow 1951), como Lavatera mauritanica Dur. subsp. davaei (Cout.) Cout., Lobularia maritima (L.) Desv. subsp. columbretensis R. Fern., Mesembryanthemum nodiflorum L., Erodium chium (L.) Willd. o Chenopodium murale L. Aparece bien representado en las islas Grossa, Mancolibre y Ferrera, conformando parte de la cubierta vegetal arbustiva de estas islas. Bolòs (1989) reconoció dos variantes de la asociación dependientes del grado de nitrificación del suelo. La variante de Lavatera arborea L. se instala sobre suelos pobres en materia orgánica (siendo propia de la isla Ferrera), mientras que la variante típica, más nitrófila (propia de la isla Grossa), queda caracterizada por Lavatera mauritanica subsp. davaei. El análisis de los inventarios originales, junto con los aquí presentados, parece indicar que ambas variantes poseen muy poco valor sintaxonómico, siendo únicamente el reflejo de la diversidad ecológica 
Tabla 2.

Mercuriali ambiguae-Succowietum balearicae O. Bolòs, Folch \& Vigo in O. Bolòs 1989 corr. Juan \& M.B. Crespo hoc loco

(Parietarion lusitanico-mauritanicae, Geranio-Cardaminetalia hirsutae, Geranio-Cardaminetea)

\begin{tabular}{|c|c|c|c|c|}
\hline Inventario $\mathrm{n}^{\circ}$ & 1 & 2 & 3 & 4 \\
\hline Orientación e inclinación $\left({ }^{\circ}\right)$ & $50 \mathrm{~N}$ & $45 \mathrm{~N}$ & $5 \mathrm{~N}$ & $4 \mathrm{~N}$ \\
\hline Altitud $(\mathrm{m})$ & 58 & 55 & 60 & 55 \\
\hline Cobertura $(\%)$ & 60 & 70 & 60 & 60 \\
\hline Área $\left(\mathrm{m}^{2}\right)$ & 7 & 10 & 10 & 10 \\
\hline Fecha (mes/año) & $3 / 96$ & $3 / 94$ & $3 / 97$ & 1979 \\
\hline \multicolumn{5}{|l|}{ Caract. de asociación y alianza } \\
\hline Mercurialis ambigua & 3 & 2 & 2 & 2.2 \\
\hline Succowia balearica & 2 & 2 & 2 & 3.2 \\
\hline Urtica membranacea & - & - & 1 & - \\
\hline \multicolumn{5}{|l|}{ Caract. de unidades superiores } \\
\hline Erodium chium & - & - & 2 & - \\
\hline Sonchus asper & - & - & + & - \\
\hline \multicolumn{5}{|l|}{ Compañeras } \\
\hline Brachypodium distachyon & 1 & + & 1 & + \\
\hline Parietaria judaica & 1 & 1 & - & 1.2 \\
\hline Sonchus dianae & + & + & + & - \\
\hline Bromus hordeaceus & 1 & - & - & - \\
\hline Fumaria munbyi & 1 & - & - & - \\
\hline Silene latifolia & - & - & 1 & - \\
\hline
\end{tabular}

Localidades: 1.- Piedras sueltas detrás del Faro, cerca del punto geodésico, ladera septentrional, Grossa. 2.- Cerca del Faro, ladera N, Grossa. 3.- Proximidades del muro del Faro, Grossa. 4.- Illa Grossa, prop del Far (Holótipo: Bolòs, 1989).

existente en el archipiélago.

En la publicación original, Bolòs (1989: 121) menciona la nueva asociación como "Lavatero mauritanicae-Suaedetum verae". Sin embargo, en la tabla de inventarios y consecuentemente en el holótipo (invent. 9), el taxon que se indica es Lavatera mauritanica subsp. davaei, por lo que creemos que el nombre que debe darse a la asociación es Lavatero davaei-Suaedetum verae, no siendo tampoco necesarias correcciones nomenclaturales. Por otro lado, la validación que proponen RivasMartínez et al. (2001), basándose en el artículo 5 del ICBN (Weber et al., 2000) no es necesaria, dado que la indicación explícita del holótipo se realizó en la tabla original, justo a continuación de la localidad del inventario 9.

\section{Mercuriali ambiguae-Succowietum balearicae}

O. Bolòs, Folch \& Vigo in O. Bolòs 1989 corr. Juan \& M.B. Crespo hoc loco

(Merculiari annuae-Succowietum balearicare O. Bolòs, Folch \& Vigo in $\mathrm{O}$. Bolòs 1989, Folia. Bot. Misc. 6: 125. Holotypus: Tabla 5, invent. 4). (Tabla 2)

Herbazal escio-nitrófilo de fenología primaveral, que se caracteriza por los táxones anuales Mercurialis ambigua L. fil. (- M. апnиa auct.) y Succowia balearica (L.) Medik., que 
Tabla 3

Withanio frutescentis-Lycietum intricati Alcaraz et al. 1991

atriplicetosum glaucae Juan \& M.B. Crespo, subass. nova (típica)

lobularietosum columbretensis Juan \& M.B. Crespo, subass. nova

\begin{tabular}{|c|c|c|c|c|}
\hline Inventario $\mathrm{n}^{\circ}$ & 1 & 2 & 3 & 4 \\
\hline Exposición e inclinación $\left({ }^{\circ}\right)$ & $25 \mathrm{E}$ & 40 & - & $25 \mathrm{~W}$ \\
\hline Altitud (m) & 40 & 35 & 5 & 35 \\
\hline Cobertura (\%) & 70 & 80 & - & 80 \\
\hline Área $\left(m^{2}\right)$ & 50 & 25 & 100 & 50 \\
\hline Fecha (mes/año) & $4 / 94$ & $4 / 94$ & - & $9 / 92$ \\
\hline \multicolumn{5}{|c|}{ Caract. de asociación y alianza } \\
\hline $\begin{array}{l}\text { Lycium intricatum } \\
\text { Withania frutescens }\end{array}$ & $\begin{array}{l}2 \\
1\end{array}$ & $\begin{array}{l}1 \\
3\end{array}$ & $\begin{array}{l}3 \\
1\end{array}$ & $\begin{array}{l}3.3 \\
3.3\end{array}$ \\
\hline $\begin{array}{l}\text { Caract. subass. atriplicetost } \\
\text { Fagonia cretica } \\
\text { Salsola oppositifolia } \\
\text { Atriplex glauca }\end{array}$ & - & - & $\begin{array}{l}+ \\
- \\
+\end{array}$ & $\begin{array}{c}1.1 \\
1.2 \\
-\end{array}$ \\
\hline $\begin{array}{l}\text { Caract. subass. lobularietos } \\
\text { Lavatera davaei } \\
\text { Lobularia columbretensis }\end{array}$ & $\begin{array}{l}1 \\
1\end{array}$ & $\begin{array}{l}1 \\
1\end{array}$ & - & - \\
\hline \multicolumn{5}{|c|}{ Caract. de unidades superiores } \\
\hline $\begin{array}{l}\text { Suaeda vera } \\
\text { Ballota hirsuta }\end{array}$ & $\begin{array}{l}3 \\
-\end{array}$ & $\begin{array}{l}1 \\
-\end{array}$ & $\begin{array}{l}1 \\
+\end{array}$ & $\begin{array}{l}- \\
+\end{array}$ \\
\hline \multicolumn{5}{|l|}{ Compañeras } \\
\hline $\begin{array}{l}\text { Lepidium graminifolium } \\
\text { Piptatherum miliaceum }\end{array}$ & - & - & + & $\begin{array}{c}1.1 \\
-\end{array}$ \\
\hline
\end{tabular}

Compañeras presentes en un solo inventario: En inv. 1: Asparagus horridus 1. En inv. 2: Brachypodium distachyon 1. En inv. 3: Convolvulus althaeoides + . En inv. 4: Urginea maritima 1.1; Opuntia ficusbarbarica +.2 .

Localidades: 1.- Ladera S del Faro, Grossa. 2.- Ladera del Faro, Grossa (holótipo subass. lobularietosum). 3.- Isla de Tabarca, Santa Pola, Alicante (holótipo subass. atriplicetosum; AlCARAZ et al., 1991). 4.- I. Benidorm, Alicante, YH5065 (Solanas, 1997).

pueden estar acompañados de otros elementos nitrófilos como Erodium chium, Urtica membranacea Poir. e incluso especies perennes nitrófilas como Parietaria judaica. Ecológicamente, esta comunidad vegetal coloniza los suelos pedregosos más o menos móviles de las zonas más umbrosas de la isla Grossa. De hecho, los mejores representantes de este sintaxon se encuentran en las laderas de orientación septentrional del Faro.

Tras la última revisión del género Mercurialis L. (Güemes, 1997), el taxon del grupo de M. annua presente en las Columbretes es realmente $M$. ambigua (ABH 15464, 15494, 44578). Por ello, y de acuerdo con el art. 43 del ICBN (op. cit.), el nombre de la asociación debe corregirse como aquí se indica.

Esta asociación también se halla presente 
en algunos pequeños islotes y cabos calcáreos de la provincia de Alicante (e.g., Escull del Cap de Sant Antoni, Calpe; Cabo de Santa Pola, etc.).

\section{Withanio frutescentis-Lycietum intricati} Alcaraz, P. Sánchez, De la Torre, Ríos \& J. Álvarez 1991

[Datos sobre la vegetación de Murcia: 84, invent. único]

lobularietosum columbretensis Juan \& M.B. Crespo, subass. nov.

(Tabla 2, holotypus: invent. 2)

Matorral halonitrófilo que coloniza acantilados rocosos y pequeños islotes, bajo bioclima termomediterráneo semiárido-seco, y cuyo óptimo corológico es murcianoalmeriense, alpujarreño-gadorense y malacitano-almijarense (cf. Alcaraz et al., 1991). En las islas Columbretes, se han localizado fragmentos residuales de esta asociación en la ladera sur del Faro y del Monumento de la isla Grossa, donde las condiciones ambientales son similares a las que se presentan en territorios más meridionales. La presencia de Withania frutescens (L.) Pauquy y Lycium intricatum Boiss., que forman pequeñas manchas, permiten su inclusión en esta asociación sin problemas.

En Columbretes, la Withanio-Lycietum se enriquece con elementos subnitrófilos, como el endemismo Lobularia maritima subsp. columbretensis y Lavatera mauritanica subsp. davaei, que permiten distinguir la nueva subasociación lobularietosum columbretensis, hasta el momento exclusiva de este archipiélago. Catenalmente, ésta establece contacto con las asociaciones Medicagini citrinae-Lavateretum arboreae O. Bolòs, Folch \& Vigo in O. Bolòs \& Vigo 1984 y Euphorbio terracinaeLobularietum columbretensis Carretero \& Boira 1987 corr. Carretero \& Aguilella 1995; siendo ambos sintáxones igualmente endémicos de este peculiar archipiélago.
Con el fin de comparar florísticamente el nuevo sintaxon con la subasociación típica (atriplicetosum glaucae Juan \& M.B. Crespo, subass. nov. Holotypus: Alcaraz \& al. 1991, loc. cit. inventario único), en la tabla 3 se han incluido dos inventarios correspondientes a esta última, procedentes de islotes de la provincia de Alicante: Tabarca, Santa Pola (inv. 3; holótipo de la subasociación atriplicetosum glaucae) e isla de Benidorm (inv. 4), en los que intervienen otros elementos diferenciales territoriales del sintaxon meridional, como Fagonia cretica y Salsola oppositifolia.

\section{ESQUEMA SINTAXONÓMICO}

PEGANO HARMALAE-SALSOLETEA VERMICULATAE Br.-B1. \& O. Bolòs 1958

+ Salsolo vermiculatae-Peganetalia harmalae Br.-Bl. \& O. Bolòs 1954

* Carthamo arborescentis-Salsolion oppositifoliae Rivas Goday \& Rivas-Martínez 1963

1. Lavatero davaei-Suaedetum verae $\mathrm{O}$. Bolòs, Folch \& Vigo in O. Bolòs 1989. [Matorral nitro-halófilo de islotes termomediterráneos]

2. Withanio frutescentis-Lycietum intricati Alcaraz, P. Sánchez, De la Torre, Ríos \& J. Álvarez 1991. [Matorral aero-halófilo de laderas rocosas y acantilados marinos termomediterráneos]

atriplicetosum glaucae Juan \& M.B. Crespo, subass. nova (típica). [variante murciano-almeriense, alpujarreñogadorense y malacitano-almijarense]

lobularietosum columbretensis Juan \& M.B. Crespo, subass. nova. [variante valenciano-tarraconense]

GERANIO PURPUREI-CARDAMINETEA HIRSUTAE (Rivas-Martínez, FernándezGonzález \& Loidi 1999) Rivas-Martínez, Fernández-González, Loidi, Lousa \& Penas 2001 
+ Geranio purpurei-Cardaminetalia hirsutae Brullo in Brullo \& Marcenò 1985

* Parietarion lusitanico-mauritanicae RivasMartínez, Fernández-González, Loidi, Lousa \& Penas 2001

\section{Mercuriali ambiguae-Succowietum} balearicae $\mathrm{O}$. Bolòs, Folch \& Vigo in $\mathrm{O}$. Bolòs 1989 corr. Juan \& M.B. Crespo hoc loco [Herbazal escio-nitrófilo de áreas iberolevantinas y murciano-almerienses]

AGRADECIMIENTOS. Queremos expresar nuestro sincero agradecimiento a los guardas del Parque Natural de las Islas Columbretes por su valiosa ayuda en los trabajos de campo y transporte a las diferentes islas del archipiélago, que se efectuaron durante las estancias realizadas en este enclave insular. Igualmente a Juan Jiménez y José Vicente Escobar de la Conselleria de Medio Ambiente (Generalitat Valenciana), por las facilidades ofrecidas para la realización del trabajo de campo. La Generalitat Valenciana y el Excmo. Ayuntamiento de Castellón han financiado parcialmente este trabajo.

\section{BIBLIOGRAFÍA}

ALCARAZ, F., P. SÁNCHEZ-GÓMEZ, A. DE LA TORRE, S. RÍOS y J. ÁLVAREZ -1991-Datos sobre la vegetación de Murcia (España). DMPPU, Murcia-Barcelona.

APARICIO, A., J. M. MITJAVILA, V. ARAÑA y I. M. VILLA -1991- La edad del volcanismo de las islas Columbrete Grande y Alborán (Mediterráneo occidental). Bol. Geol. Min. 102: 562-570.

BOLÒS, O. DE -1989- La vegetació d'algunes petites illes porperes a la Península Ibèrica. Fol. Bot. Misc. 6: 115-133.

GÜEMES, J. -1997-Mercurialis L. In: Castroviejo, S., C. Aedo, C. Benedí, M. Laínz, F. Muñoz Garmendia, G. Nieto Feliner \& J. Paiva (eds.), Flora iberica 8: 201-210. Real Jardín Botánico, CSIC. Madrid.

MATEO, G y M. B. CRESPO -2001-Manual para la determinación de la flora valenciana, $2^{\mathrm{a}}$ edición. Moliner 40. Valencia.
RIVAS-MARTÍNEZ, S., F. FERNÁNDEZ GONZÁLEZ, J. LOIDI, M. LOUSA \& A. PENAS -2001- Syntaxonomical checklist of vascular plant communities of Spain and Portugal to association level. Itinera Geobot. 14: 5-341.

RIVAS MARTÍNEZ, S. \& J. LOIDI -1999aBioclimatology of the Iberian Peninsula. In: Rivas-Martínez, S., J. Loidi, M. Costa, T. E. Díaz \& A. Penas (eds.), Iter ibericum A.D. MIM (Excursus geobotanicus per Hispaniam et Lusitaniam, ante XLII Symposium Societatis Internationalis Scientiae Vegetationis Bilbao mense Iulio celebrandum dicti Anni). Itinera Geobot. 13: 41-47.

RIVAS MARTÍNEZ, S. \& J. LOIDI -1999bBiogeography of the Iberian Peninsula. In: RIVAS-MARTÍNE Z, S., J. LOIDI, M. COSTA, T. E. DÍAZ \& A. PENAS (eds.), Iter ibericum A.D. MIM (Excursus geobotanicus per Hispaniam et Lusitaniam, ante XLII Symposium Societatis Internationalis Scientiae Vegetationis Bilbao mense Iulio celebrandum dicti Anni). Itinera Geobot. 13: 49-67.

SOLANAS, J. L. -1997-Flora, vegetació $i$ fitogeografia de la Marina Baixa. Publ. Universidad de Alicante.

WEBER, H. E., J. MORAVEC \& J. P. THÉURILLAT -2000- International Code of Phytosociological Nomenclature, 3rd edition. J. Veg. Sci. 11(5): 739-768.

Aceptado para su publicación en septiembre de 2001

Dirección de los autores. Centro Iberoamericano de la Biodiversidad (CIBIO), Universidad de Alicante, Apartado 99, E-03080 Alicante. Correo-e: ana.juan@carn.ua.es; crespo@carn.ua.es 